\title{
Intermediate Monocytes Lead to Enhanced Myocardial Remodelling in STEMI Patients With Diabetes
}

\author{
Wenbin Lu, ${ }^{1,2^{*}} \mathrm{MD}$, Ziwei ZHANG, ${ }^{2 *} \mathrm{MD}$, Cong Fu, ${ }^{1} \mathrm{MD}$, and Genshan MA, ${ }^{1} \mathrm{PhD}$
}

\begin{abstract}
SUMMARY
This study aimed to evaluate the potential associations of intermediate monocytes $\left(\mathrm{CD} 14^{++} \mathrm{CD} 16^{+}\right)$with myocardial remodelling in ST segment elevation myocardial infarction (STEMI) patients with diabetes.

A total of 67 STEMI patients with diabetes were enrolled. The control group consisted of 65 STEMI patients without diabetes. All patients received emergency medical services for reperfusion therapy in less than 12 hours after onset of AMI. Blinded to patient clinical characteristics, monocyte subset analysis was performed using flow cytometry immediately after admission. mRNA of Chemokine Decoy Receptor D6 in each subset of monocytes was validated by Q-PCR. Expression of CCL2 in patient plasma was determined with an Elisa kit. Infarct size and left ventricular ejection fraction (LVEF) were measured using 3-dimensional echocardiography 3 days and 6 months after AMI. The incidences of recurrent cardiovascular events and death in each group were measured using the Kaplan-Meier estimator in follow-up during the next 24 months. Cox proportional-hazard models were further used to analyze the relationship of monocyte cell counts and event-free survival after adjusting for confounding factors.

The number of circulating intermediate monocytes was significantly correlated with LVEF\% and infarct size $(r=$ $-0.32 ; P=0.008 ; r=0.57, P<0.001)$ in STEMI patients with diabetes compared with those without diabetes 6 months after AMI. Chemokine Decoy Receptor D6 transcript levels were lower in intermediate monocytes of STEMI patients with diabetes compared to the subsets in STEMI patients without diabetes $(P<0.001)$. Higher levels of CCL2 (pg/mL) were observed in STEMI patients with diabetes compared to STEMI patients without diabetes $(P<0.001)$. During a mean follow-up period of $24 \pm 1$ month, recurrent cardiovascular events or death occurred in 23 patients belonging to the STEMI with diabetes group and 10 belonging to the control group. Univariate Kaplan-Meier analysis revealed that counts of the intermediate monocytes according to median showed statistical significance in STEMI patients with diabetes $(P=0.010)$. After full adjustment for confounding factors, the cells were found to remain independently related to recurrent cardiovascular events or death in this group $(P=0.004,95 \% \mathrm{CI}$ : $1.62-12.49)$.

Intermediate monocytes were associated with LV remodelling in STEMI patients with diabetes. The cells were predictive for recurrent cardiovascular events or death in these patients. A low level of D6 mRNA in the intermediate monocytes of STEMI patients with diabetes and high level of CCL2 in these patients may partially explain the causality. (Int Heart J 2015; 56: 22-28)
\end{abstract}

Key words: CD14, CD16, Myocardial infarction

$\mathrm{A}$ cute myocardial infarction (AMI), which is an acute pathology associated with atherosclerotic plaque rupture and interruption of coronary blood supply to myocardial tissue, is the most frequent cause of cardiac death. The involvement of inflammation in the pathophysiology of AMI has been identified in recent years, ${ }^{1,2)}$ and monocytes/ macrophages were found to be the key players in this process. ${ }^{3,4)}$ The ability of monocytes to differentiate into various cell phenotypes suggests remarkable "plasticity" in response to AMI. The prevailing belief is that circulating monocytes are relatively uncommitted and that their eventual phenotype entirely depends on the tissue environment. ${ }^{5)}$ In mice, one subset of monocytes (Ly-6C $\mathrm{C}^{\mathrm{hi}}$ ) aggravates inflammation and expands in AMI conditions, whereas another subset of monocytes (Ly$6 \mathrm{C}^{\text {low }}$ ) attenuates inflammation and promotes angiogenesis and granulation tissue formation in the process. In humans, monocytes have recently been defined and divided into three subsets ${ }^{6,7)}$ : (1) "classical" monocytes $\left(\mathrm{CD} 14^{++} \mathrm{CD} 16^{-}\right.$; also referred to as Mon1), which account for $80 \%$ to $85 \%$ in the total population of circulating monocytes; (2) $\mathrm{CD} 14^{+} \mathrm{CD} 16^{++}$subset referred to as "nonclassical" monocytes or Mon3 population; and (3) "intermediate" subsets of monocytes $\left(\mathrm{CD} 14^{++} \mathrm{CD} 16^{+}\right)$ confirmed recently by gene microarray analysis and referred to as Mon2. ${ }^{8)}$ Compared with the other two subsets, the interme-

From the ${ }^{1}$ Department of Cardiology, ZhongDa Hospital Affiliated to Southeast University China and ${ }^{2}$ Division of Endocrinology, The Drum Tower Hospital Affiliated to Nanjing University, Nanjing, China.

"These authors contributed equally to this work

This work was supported by the National Natural Science Foundation of China (No. 81300160) and LJ 201146.

Address for correspondence: Genshan Ma, PhD, Department of Cardiology, ZhongDa Hospital Affiliated to Southeast University China, 87\#, Dingiaqiao Road, Nanjing 210009, China. E-mail: magenshanseu@163.com

Received for publication May 30, 2014. Revised and accepted June 16, 2014.

Released in advance online on J-STAGE December 11, 2014.

All rights reserved by the International Heart Journal Association. 
diate $\mathrm{CD} 14^{++} \mathrm{CD} 16^{+}$monocyte subsets are poorly understood and characterized. Recent findings suggest that Mon2 is important in the pathogenesis of cardiovascular diseases and exhibits proinflammatory and proatherosclerotic activities in in vitro experiments. ${ }^{9)}$ In this study, we assessed the association of $\mathrm{CD} 14^{++} \mathrm{CD} 16^{+}$monocyte subsets with myocardial impairment in vivo after AMI in STEMI patients with diabetes.

\section{Methods}

Study population: The participants were less than 80 years old with or without diabetes, who were admitted to Medical Center of Zhongda hospital affiliated to Southeast University for AMI from 2009 to 2010. All patients received emergency medical services for reperfusion therapy within 12 hours after the onset of AMI. Lesion vessels and management for AMI patients in the two groups were listed in Supplemental Table I. This study was approved by the local ethics committee, and all participants provided written informed consent. For patients who had acute ST segment elevation myocardial infarction (STEMI), at least one of the following two criteria had to be met in addition to persistent ST-segment elevation more than $0.1 \mathrm{mV}$ in at least two contiguous leads: a positive biomarker test (TnI) indicating myocardial necrosis; and persistent symptoms of chest pain or tightness for myocardial ischemia. Eligible STEMI patients with self- or physician-reported diabetes mellitus, non-fasting blood glucose levels $\geq 11.1 \mathrm{mmol} / \mathrm{L}$ and fasting blood glucose levels $\geq 7.0 \mathrm{mmol} / \mathrm{L}$, or current use of hypoglycemic medication were categorized as having diabetes. Subjects showing any of the following exclusion criteria were not allowed to participate in the study: $\geq 80$ years old or $<18$ years old; with severe liver or kidney diseases; with glomerular filtration rate $(\mathrm{GFR})$ of $<30 \mathrm{~mL} /\left(\right.$ minute $1.73 \mathrm{~m}^{2}$ ) or ChildTurcotte-Pugh (CTP) score $\geq 6$; with hemodynamic or electrical instability (including shock); coagulation disorders associated with significant bleeding tendency; a platelet count of less than $100 \times 10^{9} / \mathrm{L}$ or hemoglobin less than $100 \mathrm{~g} / \mathrm{L}$; intake of systemic immunosuppressive agents; occurrence of other serious disorders; life expectancy of less than 6 months. The basic characteristics of patients were acquired by a questionnaire and clinical examination, which included a history of cardiovascular or cerebrovascular diseases, smoking, drug intake, body mass index, blood pressure, and GFR. Primary endpoint was defined as death due to cardiovascular or vascular causes and recurrent cardiovascular events (re-PCI, re-myocardial infarction, stent thrombosis, and stroke), all of which were in accordance with the Academic Research Consortium criteria.

Laboratory measurements: Blood samples were obtained im- mediately upon admission. Serum levels of troponin (TnI), myoglobin, creatine kinase $\mathrm{MB}$, and brain natriuretic peptide were determined in 24 hours after the onset of AMI. Fasting levels of creatinine, total cholesterol, low-density lipoprotein (LDL) cholesterol, high-density lipoprotein cholesterol, and Creactive protein (CRP) were measured using standard methods at the laboratory of Zhongda Hospital.

Mononuclear cells were initially acquired by centrifuging $5 \mathrm{~mL}$ of heparinized blood on a Hypaque-Ficoll density gradient at $1250 \times \mathrm{g}$ for 20 minutes. Isolated mononuclear cells were then incubated with PerCP/Cy5.5-conjugated monoclonal antibodies against the molecule CD14 (Becton Dickinson, USA) and with Alexa Fluor conjugated monoclonal antibodies against the molecule CD16 (Becton Dickinson, USA). After preparation, flow cytometry analysis was performed with a FACSCalibur flow cytometer (Becton Dickinson). Briefly, monocytes were gated in a side scatter and then subpopulations of $\mathrm{CD} 14^{++} \mathrm{CD} 16^{-}, \mathrm{CD} 14^{++} \mathrm{CD} 16^{+}$, and $\mathrm{CD} 14^{+} \mathrm{CD} 16^{++}$ monocytes were distinguished by their surface expression pattern of CD14 and the Fc $\gamma$ IIIR CD16. The representative process of the distribution of the subpopulations of CD14 or CD16 monocytes in the peripheral blood of AMI patients is shown in Figure 1.

Quantitative PCR and Elisa: Quantitative Reverse Transcrip-
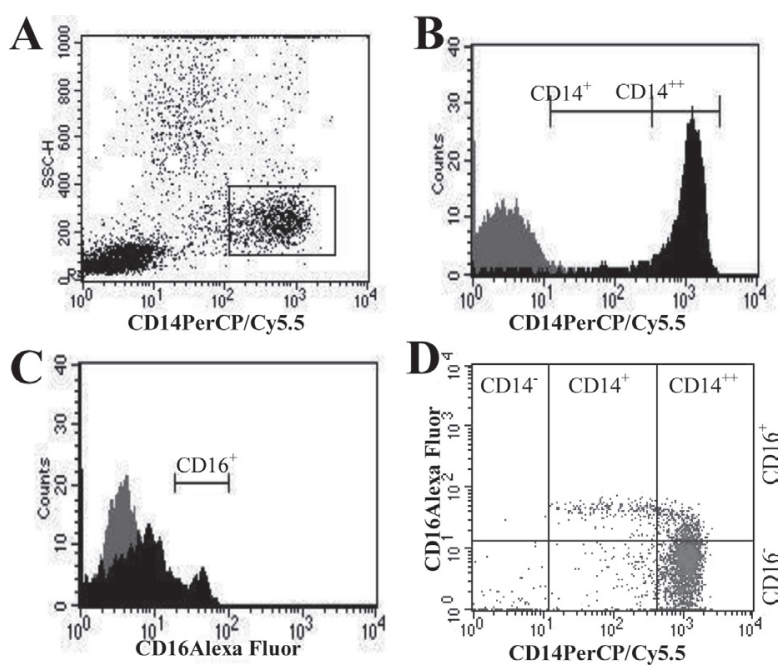

Figure 1. Representative images of analyzing monocyte subsets in patient blood by flow-cytometry. CD14 monocytes were first gated using an SSCheight/CD14-PerCP/Cy5.5 dot plot (A). Different expressions of CD14 and CD16 are shown in $\mathbf{B}$ and $\mathbf{C}$. The monocyte subsets population within A was assessed using CD16-Alexa Fluor and CD14-PerCP/Cy5.5 dot plot (D).

Table I. Primary Endpoint in Two Groups of Patients

\begin{tabular}{lccc}
\hline Endpoint & $\begin{array}{c}\text { STEMI with diabetes } \\
(n=67)\end{array}$ & $\begin{array}{c}\text { STEMI without diabetes } \\
(n=65)\end{array}$ & $P$ \\
\hline re-PCI & $12(17.9 \%)$ & $5(7.7 \%)$ & 0.080 \\
stent thrombosis & $1(1.5 \%)$ & $0(0.0 \%)$ & 0.508 \\
re-myocardial infarction & $3(4.5 \%)$ & $1(1.5 \%)$ & 0.619 \\
nonhemorrhagic stroke & $3(4.5 \%)$ & $2(3.1 \%)$ & 0.514 \\
cardiovascular or vascular death, & $4(6.0 \%)$ & $2(3.1 \%)$ & 0.355 \\
Total, $n(\%)$ & $23(34.3 \%)$ & $10(15.4 \%)$ & 0.012 \\
\hline
\end{tabular}

Endpoints after 24 months of follow-up. PCI indicates percutaneous coronary intervention. 
tion Polymerase Chain Reaction: Total RNA was extracted from each subset of monocytes isolated with a FACSCalibur flow cytometer with Trizol reagent according to the manufacturer's protocol (Invitrogen). QuantiTect Reverse Transcription Kit (Fermentas) was used for reverse transcrition. Polymerase chain reaction was performed by Platinum SYBR Green qPCR SuperMix UDG (Applied Invitrogen) in a BIO-RAD MJ Mini Opticon Real-Time PCR System. Primer sequences were as follows: human D6 (Gene Bank Y12815.1 110 bytes), 5-AGGAAGGATGCAGTGGTGTC-3 and 5-CGGAGCAAGACCATGAGAAG-3; human GAPDH, 5-CAAGGCTGAGAACGGGAAG-3 and 5-GGTGGTGAAGACGCCAGT-3;

Chemokine levels of CCL2 in the serum were measured using the human ELISA Ready-SET-Go kit (R\&D Systems Inc.) according to the manufacturer's protocol. The absorbance of the test sample was compared with the standard curve. The concentrations were determined in duplicate according to the manufacturer's protocol.

Measurement of infarct size and left ventricular ejection fraction (LVEF): Three-dimensional (3D) echocardiography using GE vivid E9 with a novel 3V matrix-array transducer was performed by rotational image acquisition from a parasternal view. ${ }^{10)} 3 \mathrm{D}$ left ventricular (LV) volumes and LVEF were measured from the 3D full-volume data set using Echo Pac software. The full-volume data of the LV was divided into orthogonal 4-chamber, 2-chamber, and short-axis views. End-diastolic and end-systolic frames were selected. Semi-automated LV endocardial border detection software on Echo Pac was used to outline the endocardial borders in these 3 planes. Subsequently, the software automatically calculated true 3D enddiastolic volume, end-systolic volume, and LVEF (Figure 2). 3D wall-motion score (WMS) was analyzed in the dynamic multi-slice view to ensure continuous visualization of the same cardiac structures throughout the cardiac cycle. The slices were automatically updated after tracking in each frame to correct out-of-plane motion caused by longitudinal shortening of the LV. WMS for 3D echocardiography was assessed in a 16-segment model called Bull's-eye. Segmental WMS was graded as $1,2,3$, and 4 (normal, hypokinetic, akinetic, and dyskinetic, respectively). Wall-motion score index was calculated by averaging the scored segments when 16 segments were accepted for analysis. The Bull's-eye presentation of wall-motion scores
(WMS) indicated the segments of myocardial infarction according to the grade of the left ventricular segment.

Statistical analysis: Data management and statistical analysis were performed using SAS software version 9.1 (SAS Institute, USA). A $P<0.05$ was considered statistically significant. Data are expressed as the mean \pm standard deviation or as the median and interquartile range when necessary. Categorical variables were compared using the $\chi^{2}$ test or Fisher's exact test. For continuous data, group comparisons were performed using an unpaired $t$ test or the Mann-Whitney $U$ test. Correlations between the intermediate monocytes and LVEF as well as infarct size were assessed by the Spearman rank test after log transformation. Kaplan-Meier survival curves were used to compare event-free survival (the first occurrence of the primary endpoints) between groups. Cox proportional hazards models were introduced to analyze the relationship of monocyte cell counts with event-free survival after adjusting for age and sex, body mass index, blood pressure, smoking, LDL, high-sensitivity CRP (hs-CRP), eGFR, total leukocyte count, and drugs taken (antiplatelet agents, $\beta$-blockers, ACE inhibitors/ARB, and statins).

\section{RESULTS}

Baseline characteristics of patients in the two groups: In the 24 month follow-up period, a total of 23 subjects in the STEMI with diabetes group and 10 in the STEMI without diabetes group experienced recurrent cardiovascular events or death, for a total of 33 patients who experienced a primary endpoint (Table I). The basic characteristics of the study population are shown in Supplemental Table II. No significant difference was observed between the two groups in terms of age, sex, levels of TnI, LDL, total leukocytes, previous medication, and other parameters, except for antidiabetic drugs and hemoglobin A1c. However, STEMI patients with diabetes showed slightly higher markers of hs-CRP for chronic inflammation than patients in the STEMI without diabetes group.

Comparison of the number of intermediate monocyte cells in the two groups: Three subsets of monocytes were counted by FACSC upon admission in both groups and were expressed as the percentage of positive cells (Figure 1). Each subset of
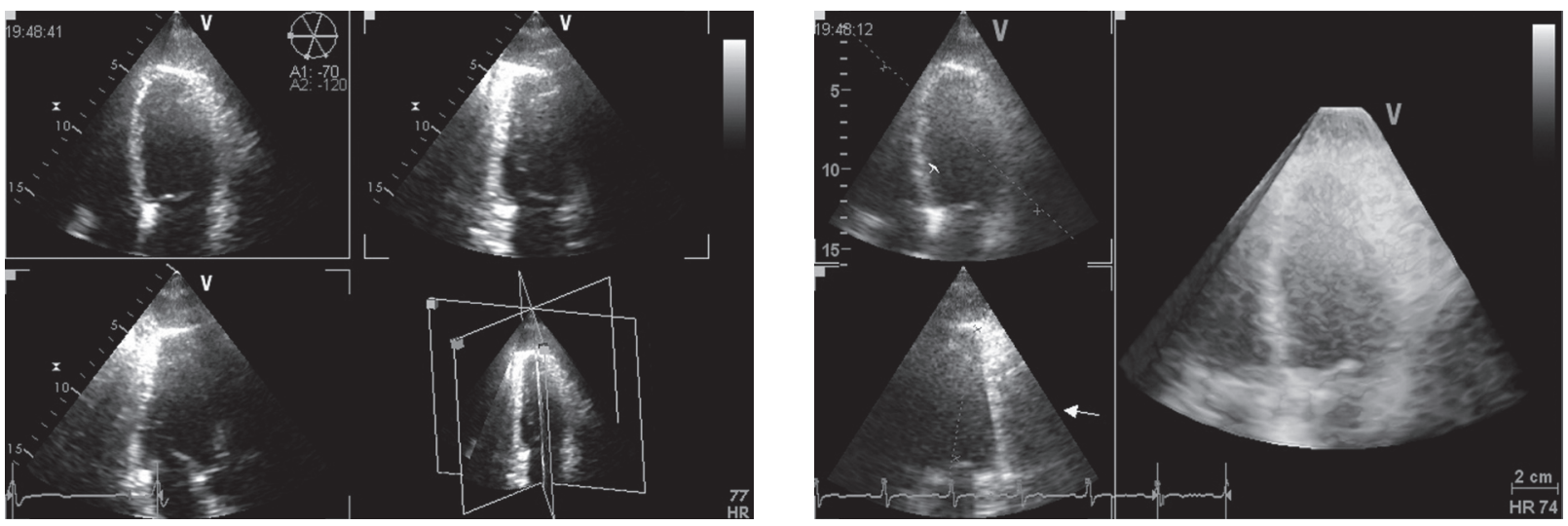

Figure 2. Presentation of left ventricle using 4D auto LVQ software for measurement of left ventricular volumes and ejection fraction with three-dimensional echocardiography. Volume time-plot and quantitative analysis and three-dimensional model are presented in the right panel. This panel demonstrates extensive apical akinesis and basal hyperkinesis of the left ventricle. 
Table II. Number and Percentage of the Three Subsets of Monocytes

\begin{tabular}{|c|c|c|c|}
\hline Monocyte subset & STEMI with diabetes & STEMI without diabetes & $P$ \\
\hline $\mathrm{CD}_{14}{ }^{++} \mathrm{CD} 6^{-}$monocytes & $80.3 \pm 14(\%)$ & $80.7 \pm 20(\%)$ & 0.577 \\
\hline $\mathrm{CD}_{14}{ }^{++} \mathrm{CD}_{16}{ }^{+}$monocytes & $4.1 \pm 1.1(\%)$ & $3.7 \pm 1.3(\%)$ & $0.015^{*}$ \\
\hline $\mathrm{CD}_{14}{ }^{+} \mathrm{CD} 16^{++}$monocytes & $7.7 \pm 2.6(\%)$ & $7.5 \pm 3.4(\%)$ & 0.282 \\
\hline $\mathrm{CD}_{14}{ }^{++} \mathrm{CD} 16^{-}$monocytes & $467 \pm 139 / \mathrm{uL}$ & $462 \pm 144 / \mathrm{uL}$ & 0.460 \\
\hline $\mathrm{CD}_{14}{ }^{++} \mathrm{CD}_{16}{ }^{+}$monocytes & $41 \pm 16 / \mathrm{uL}$ & $32 \pm 15 / \mathrm{uL}$ & $0.002^{*}$ \\
\hline $\mathrm{CD}_{14}{ }^{+} \mathrm{CD} 16^{++}$monocytes & $69 \pm 27 / \mathrm{uL}$ & $72 \pm 25 / \mathrm{uL}$ & 0.390 \\
\hline
\end{tabular}

FACSC analysis and counts of monocytes subsets. Values are mean \pm SD, STEMI indicates ST segment elevation myocardial infarction. ${ }^{*} P<0.05$ : STEMI with diabetes versus STEMI without diabetes.
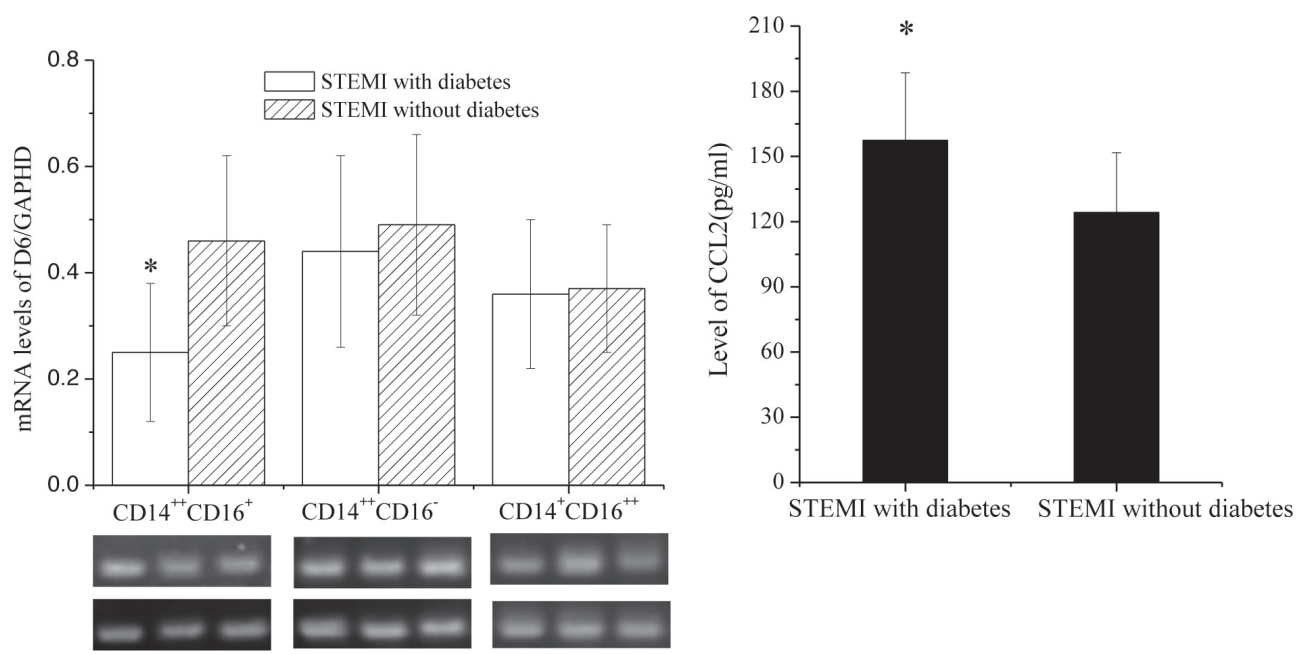

Figure 3. Analysis mRNA of Chemokine Decoy Receptor D6 in three subsets of monocytes in each group (Left); Detection Chemokine levels of CCL2 in the plasma of the two groups (Right). ${ }^{*} P<0.05$.

monocytes was further counted per microliter of whole blood. Results showed that both the number and percentage of the intermediate $\left(\mathrm{CD} 14^{++} \mathrm{CD} 16^{+}\right)$monocytes were higher in the STEMI patients with diabetes compared with the group of STEMI patients without diabetes $(4.1 \% \pm 1.1 \%$ versus $3.7 \% \pm$ $1.3 \%, P=0.015$ ) (Table II). No difference was observed in the $\mathrm{CD} 14^{++} \mathrm{CD} 16^{-}$and $\mathrm{CD} 14^{+} \mathrm{CD} 16^{++}$subsets between the two groups.

Analysis of D6 expression in human monocyte subtypes and CCL2 in the plasma: To determine whether the three subsets of monocytes are reflected in different types and extent of inflammation, we next analyzed the mRNA expression of Chemokine Decoy Receptor D6 on monocytes. In agreement with recent studies in mice, ${ }^{11,12)}$ the patient monocyte QPCR data also demonstrated expression of D6 on monocytes, although at relatively low levels. Intriguingly, as shown in Figure 3, Chemokine Decoy Receptor D6 transcript levels were lower in the intermediate monocytes of STEMI patients with diabetes compared to STEMI patients without diabetes $(0.25 \pm 0.13$ versus $0.46 \pm 0.16, P<0.001)$ and no statistical significance was observed in the other two subsets of monocytes between the STEMI patients with or without diabetes. Detection of D6 expression on monocytes prompted us to further examine the expression of CCL2 in plasma, which plays an important role in the recruitment of monocytes after AMI. As expected, the results detected by Elisa showed higher levels of CCL2 (pg/mL) expression in STEMI patients with diabetes compared to STEMI patients without diabetes $(157.35 \pm 31.24$ versus $124.2 \pm$
27.5, $P<0.001)$.

Association of the intermediate monocytes and myocardial remodelling in two groups: Three days and 6 months after the onset of AMI, patient LVEF and infarct size were acquired by 3D echocardiography. ${ }^{13,14)}$ We did not observe significant differences regarding LVEF or infarction size (\%) between the groups at these two time points, although a slight difference in infarction size was observed in STEMI patients with diabetes compared to the control at 3 days $(0.50 \pm 0.15$ versus $0.52 \pm$ $0.22, P=0.59)$ and at 6 months $(0.36 \pm 0.11$ versus $0.34 \pm$ $0.15, P=0.42$ ) (Supplemental Figure 1). However, there was a significant difference in the reduction of the infarction size between STEMI patients with diabetes and the control group at 6 months $(0.14 \pm 0.06$ versus $0.18 \pm 0.09, P<0.001)$. Additionally, as shown in Supplemental Figure 2, the intermediate monocytes showed a significant association with LVEF at 6 months in STEMI patients with diabetes $(r=-0.32 ; P=0.008)$ but not in the control group of STEMI without diabetes $(r=$ $-0.17 ; P=0.18$ ). A significantly positive correlation between the cells and infarct size was also observed in STEMI patients with diabetes $(r=0.57 ; P<0.001)$ compared with the control group $(r=0.07 ; P=0.57)$ at 6 months.

Association of $\mathrm{CD}^{++} \mathrm{CD}^{+}{ }^{+}$monocytes and cardiovascular outcomes in STEMI patients with or without diabetes: As shown in Table IV, STEMI patients with diabetes had a mean of $41 \pm 16 / \mu \mathrm{L}$ intermediate monocytes compared with $32 \pm 15$ / $\mu \mathrm{L}$ in STEMI patients without diabetes $(P=0.002) ; 467 \pm 139$ CD $14{ }^{++}$CD $16^{-}$"classical" monocytes $/ \mu \mathrm{L}$ and $69 \pm 27$ 
$\mathrm{CD} 14^{+} \mathrm{CD} 16^{++}$"nonclassical" monocytes $/ \mu \mathrm{L}$ corresponding to $462 \pm 144 / \mu \mathrm{L}(P=0.46)$ and $72 \pm 25 / \mu \mathrm{L}(P=0.39)$ in STEMI patients without diabetes, respectively. Study cohorts were then stratified according to the median of intermediate monocyte counts in each group. Survival curves showed that a higher level of intermediate monocytes was univariately associated with the endpoint defined above in STEMI patients with diabetes but not in the STEMI without diabetes group. Multivariate regression analysis was further applied after adjustment for age, sex, body mass index, blood pressure, smoking, LDL, hsCRP, eGFR, total leukocyte count, and drugs taken (antiplatelet agents, $\beta$-blockers, ACE inhibitors/ARB, and statins). Subsequently, the "intermediate" CD14 ${ }^{++} \mathrm{CD} 16^{+}$subset in STEMI patients with diabetes still predicted adverse cardiovascular outcomes $(P=0.004,95 \%$ CI: $1.62-12.49)$. The survival function at the mean of covariates is shown in Figure 4.

\section{Discussion}

Acute myocardial infarction (AMI) is an acute inflammatory disease characterized by the rupture of arterial lesions and the interruption of blood supply to the myocardium. This phenomenon is followed by the infiltration of circulating monocytes to nascent lesions under the attraction of pro-inflammatory molecules secreted by endothelial cells. The central role of circulating monocytes in the development and progression of AMI has been experimentally confirmed by knockout animal studies. ${ }^{15-17)}$ Recently, an updated classification of monocyte heterogeneity following the differentiation of CD16-positive monocytes into $\mathrm{CD} 14^{++} \mathrm{CD} 16^{+}$and $\mathrm{CD} 14^{+} \mathrm{CD} 16^{++}$monocytes has been established, and the existence of 3 monocyte subsets has been acknowledged. ${ }^{18)}$ However, the intermediate monocyte subset remains poorly characterized because most clinical and experimental researchers assume that the intermediate and nonclassical monocytes are a single subset. Evidence indicates that intermediate monocytes are important in the pathology of cardiovascular diseases. ${ }^{19,20)}$ The subsets show high activation and inflammatory potential by selective upregulating of genes (AIF1 and TGFB1) linked to the inflammatory processes and display the ability to produce reactive oxygen species. ${ }^{21,22)}$ Therefore, the biological behaviour of these monocytes in AMI patients with diabetes should be determined because these patients are associated with increased immediate and long-term systemic inflammation, ${ }^{23-25)}$ especially in small and large blood vessels.

In this study, we showed that the intermediate monocytes were closely related to the extent of infarction size and LVEF in STEMI patients without diabetes. Furthermore, elevated intermediate monocytes could predict cardiovascular events in these patients. This result indicated that the intermediate monocytes had different biological functionalities. We already knew that inflammatory $\mathrm{CD} 14^{++} \mathrm{CD} 16^{-}$cells quickly increased in the circulation and were recruited to injured myocardium under acute conditions such as AMI, following which they differentiated into the intermediate monocytes $\left(\mathrm{CD} 14^{++} \mathrm{CD} 16^{+}\right.$
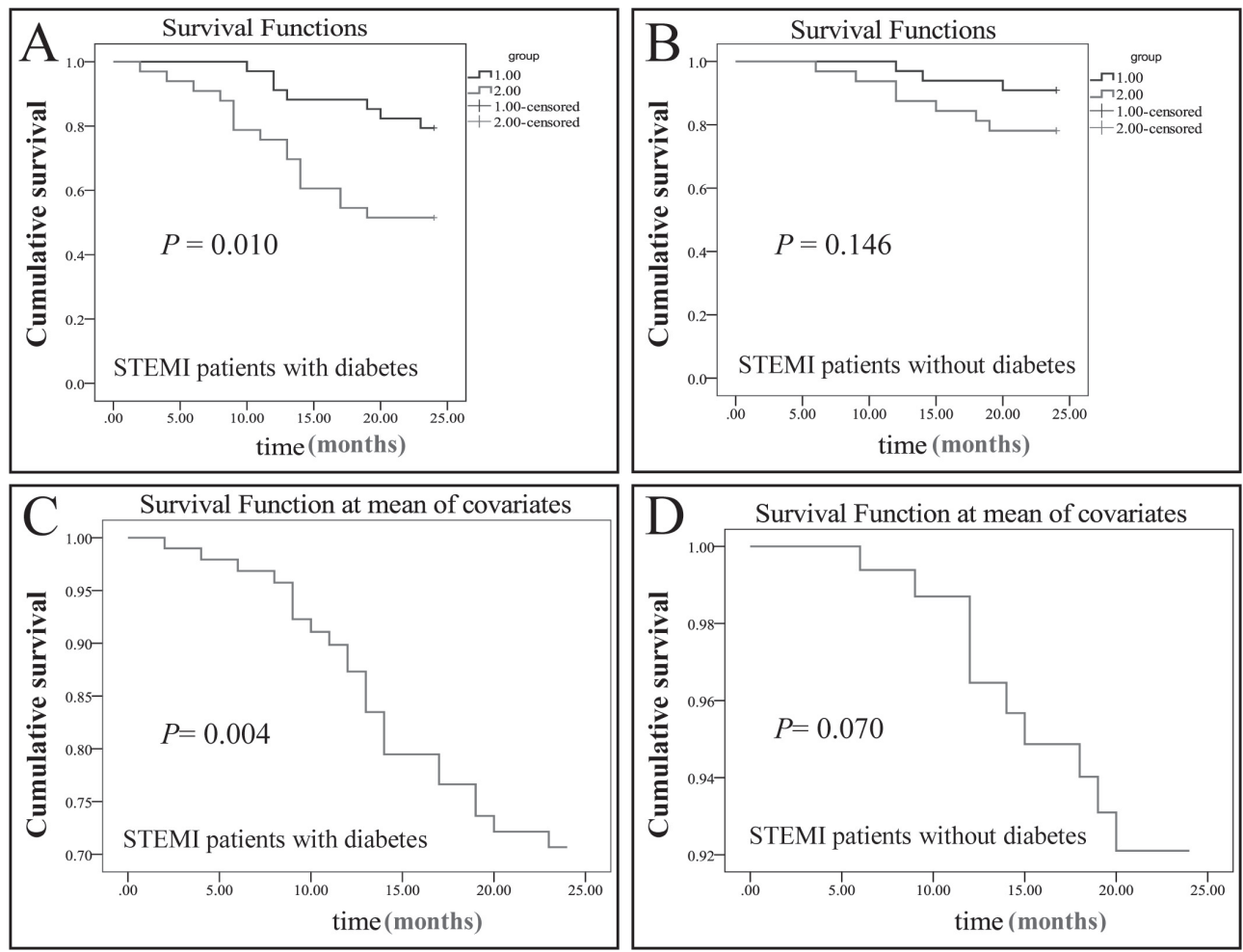

Figure 4. Univariate Kaplan-Meier and multivariate regression analysis. A: Association of the intermediate monocytes stratified according to the median and event-free survival in STEMI patients with diabetes. B: Association of the intermediate monocytes stratified according to the median and event-free survival in STEMI patients without diabetes. (blue line in A and B represents under median and red is over median); C,D: Multivariate regression analysis after adjusting for confounding factors in STEMI patients with diabetes and in STEMI patients without diabetes. 
subsets) with increasing duration. A sustained high number of intermediate monocytes in STEMI patients, especially those with diabetes, may result in ongoing inflammation infiltration, which adversely affects the rehabilitation of infarction myocardium and weakens other fibrous caps covering plaques. Thus, a sustained high number of these cells may be closely related to increased risk of cardiovascular events or death. This study is the first to show that "CD $14^{++} \mathrm{CD} 16^{+}$intermediate" subsets of monocytes negatively affected injured myocardium repair and could predict future cardiovascular risk regardless of other traditional risk factors in STEMI patients with diabetes. However, it should be noted we cannot conclude anything definitive about the association between LV function and the intermediate monocytes at the onset of AMI since we did not take into account the injured LV wall function from previous myocardial infarctions or silent myocardial ischemia with low EF\% before AMI in this study.

Our results also partially confirmed the findings of Kyrill $\mathrm{S}$, et $a l,{ }^{26)}$ who showed that $\mathrm{CD} 14^{++} \mathrm{CD} 16^{+}$monocytes independently predict cardiovascular events in subjects referred for elective coronary angiography, as well as the findings of Montange, et $a l^{27)}$ who described the effect of $\mathrm{CD} 14^{+}$monocytes on myocardial salvage and infarction size in STEMI patients. However, our results did not support the validity of these findings in all coronary artery diseases because we only examined the number of the cells in a certain population of STEMI patients with diabetes. Recent studies ${ }^{19,28}$ have mainly focused on the prominence of $\mathrm{CD}_{16}{ }^{+}$monocytes but not $\mathrm{CD} 16^{-}$monocytes in inflammatory diseases, such as AMI and diabetes. The accumulation of such cells in peripheral blood mainly functioned in inducing and perpetuating the inflammatory process in patients with acute and chronic inflammatory diseases. Some studies ${ }^{29-31)}$ have shown that $\mathrm{CD} 16^{+}$monocyte counts are elevated in many inflammatory conditions and that $\mathrm{CD}^{+} 6^{+}$ monocytes are efficient producers of inflammatory cytokines with high endothelial affinity.

It has been determined that D6 is able to bind to and cause the degradation of most inflammatory CC chemokines and does not lead to directional cell migration. Inflammatory monocytes were dramatically involved in the process of AMI and recruited under the reaction of CCL2. We postulated that expression of D6 on the monocytes might be central to its ability to scavenge inflammatory CCL2 and contribute to the resolution of local inflammatory responses after AMI. Thus, we set out to identify D6 mRNA from each subset of monocytes and CCL2 in plasma in the two groups of patients. We found low levels of D6 mRNA on the intermediate monocytes in STEMI patients with diabetes and increased CCL2 in these patients. This might suggest that these cells are closely related to increased inflammation and heart impairment especially in STEMI patients with diabetes since evidence obtained under homeostatic conditions ${ }^{32)}$ suggests that D6-deficient mice present a selective increase of spleen Ly6 $\mathrm{C}^{\text {high }}$ monocytes in the circulation and develop exacerbated inflammatory responses in different experimental diseases sustained by increased levels of inflammatory $\mathrm{CC}$ chemokines. Taken together, this may partially explain why we found a negative association between the percentage of intermediate monocytes and STEMI patients with diabetes, but not STEMI patients without diabetes.

This result was in accordance with recent studies ${ }^{33-35)}$ in patients on dialysis and with chronic kidney disease, which suggested an elevated risk of cardiovascular events when in the presence of a higher level of the intermediate monocytes. This finding also indicated that traditional risk factors accounted for only a small fraction of the excess risk. Therefore, our findings that the percentage of intermediate monocytes was negatively associated with myocardium rehabilitation and can predict elevated risk of cardiovascular events may account for non-traditional risk factors, such as the immune system, which functions in the long-term process. Studies ${ }^{9,36,37)}$ have demonstrated that intermediate monocytes differentiate in vitro in a Th2-dependent manner and display the characteristics of senescent cells with increased $\beta$-galactosidase concentration and shortening of telomeres. These previous results suggest that the Th2-dependent environment in patients with chronic inflammatory disease may enhance the survival of activated monocytes in the event of acute diseases and thus become senescent. In the end, the cells were associated with an increased risk of cardiovascular events and indicated disease progression.

However, the present study did not prove causality, it merely detected associations. We cannot prove that the high intermediate monocyte counts caused the initiation and progression of AMI or the subsequent processing in STEMI patients with diabetes. In addition, we cannot exclude the possibility that the intermediate monocytes may reflect a downstream event from the baseline. Furthermore, 3-dimensional echocardiography was introduced for measurement of infarct size in STEMI patients. Most likely it can not offer a very high sensitivity and specificity compared to magnetic resonance imaging (MRI). The methods adopted here were chosen mainly in consideration of the facility and feasibility of the study, meanwhile, some research has also suggested the accuracy ${ }^{10,38)}$ of 3-dimensional echocardiography is credible for the measurement of infarct size with a $76 \%$ sensitivity and $72 \%$ specificity compared to MRI. In the end, this study was also limited by the relatively small number of patients included.

Conclusion: The intermediate monocytes had a negative effect on myocardial remodelling in STEMI patients with diabetes. We further observed an association of the cells with cardiovascular events in the subjects and partially described the possible mechanisms of Chemokine Decoy Receptor D6 expression. However, whether the cells represent a target cell population for new therapeutic strategies in AMI and atherosclerosis should be further studied.

\section{Disclosure}

Competing interests: The authors hereby declare that no competing interests exist.

\section{REFERENCES}

1. Legein B, Temmerman L, Biessen EA, Lutgens E. Inflammation and immune system interactions in atherosclerosis. Cell Mol Life Sci 2013; 70: 3847-69. (Review)

2. Czepluch FS, Schlegel M, Bremmer F, Behnes CL, Hasenfuss G, Schäfer K. Stage-dependent detection of CD14+ and CD16+ cells in the human heart after myocardial infarction. Virchows Arch 2013; 463: 459-69.

3. Sheu JJ, Chang LT, Chiang CH, et al. Prognostic value of activated toll-like receptor-4 in monocytes following acute myocardial in- 
farction. Int Heart J 2008; 49: 1-11.

4. Charo IF. Blinding the monocytes to protect the heart. Circulation 2013; 127: 2006-8.

5. Nahrendorf M, Pittet MJ, Swirski FK. Monocytes: protagonists of infarct inflammation and repair after myocardial infarction. Circulation 2010; 121: 2437-45. (Review)

6. Zawada AM, Rogacev KS, Rotter B, et al. SuperSAGE evidence for CD14++CD16+ monocytes as a third monocyte subset. Blood 2011; 118: e50-61.

7. Cros J, Cagnard N, Woollard K, et al. Human CD14dim monocytes patrol and sense nucleic acids and viruses via TLR7 and TLR8 receptors. Immunity 2010; 33: 375-86.

8. Ghattas A, Griffiths HR, Devitt A, Lip GY, Shantsila E. Monocytes in coronary artery disease and atherosclerosis: where are we now? J Am Coll Cardiol 2013; 62: 1541-51. (Review)

9. Merino A, Buendia P, Martin-Malo A, Aljama P, Ramirez R, Carraedo J. Senescent CD14+CD16+ monocytes exhibit proinflammatory and proatherosclerotic activity. J Immunol 2011; 186: 1809-15.

10. Thorstensen A, Dalen H, Hala P, et al. Three-dimensional echocardiography in the evaluation of global and regional function in patients with recent myocardial infarction: a comparison with magnetic resonance imaging. Echocardiography 2013; 30: 682-92.

11. McKimmie CS, Fraser AR, Hansell C, et al. Hemopoietic cell expression of the chemokine decoy receptor D6 is dynamic and regulated by GATA1. J Immunol 2008; 181: 8171-81.

12. Graham GJ, Locati M. Regulation of the immune and inflammatory responses by the 'atypical' chemokine receptor D6. J Pathol 2013; 229: 168-75. (Review)

13. Vieira ML, Oliveira WA, Cordovil A, et al. 3D Echo pilot study of geometric left ventricular changes after acute myocardial infarction. Arq Bras Cardiol 2013; 101: 43-51. (English, Portuguses)

14. Lemkes J, Nijveldt R, Beek AM, et al. Evaluating the Optimal Timing of Revascularization in Patients with Transient ST-Segment Elevation Myocardial Infarction: Rationale and Design of the TRANSIENT Trial. J Cardiovasc Transl Res 2014; 7: 590-6.

15. Kamei M, Carman CV. New observations on the trafficking and diapedesis of monocytes. Curr Opin Hematol 2010; 17: 43-52. (Review)

16. Swirski FK, Weissleder R, Pittet MJ. Heterogeneous in vivo behavior of monocyte subsets in atherosclerosis. Arterioscler Thromb Vasc Biol 2009; 29: 1424-32. (Review)

17. Swirski FK, Nahrendorf M, Etzrodt M, et al. Identification of splenic reservoir monocytes and their deployment to inflammatory sites. Science 2009; 325: 612-6.

18. Ziegler-Heitbrock L, Ancuta P, Crowe S, et al. Nomenclature of monocytes and dendritic cells in blood. Blood 2010; 116: e74-80.

19. Rogacev KS, Seiler S, Zawada AM, et al. CD14++CD16+ monocytes and cardiovascular outcome in patients with chronic kidney disease. Eur Heart J 2011; 32: 84-92.

20. Heine GH, Ortiz A, Massy ZA, et al. Monocyte subpopulations and cardiovascular risk in chronic kidney disease. Nat Rev Nephrol 2012; 8: 362-9. (Review)

21. Marketou ME, Kontaraki JE, Tsakountakis NA, et al. Differential effect of telmisartan and amlodipine on monocyte chemoattractant protein-1 and peroxisome proliferator-activated receptor-gamma gene expression in peripheral monocytes in patients with essential hypertension. Am J Cardiol 2011; 107: 59-63.

22. Abeles RD, McPhail MJ, Sowter D, et al. CD14, CD16 and HLADR reliably identifies human monocytes and their subsets in the context of pathologically reduced HLA-DR expression by CD14(hi) /CD16(neg) monocytes: Expansion of CD14(hi) / CD16(pos) and contraction of CD14(lo) /CD16(pos) monocytes in acute liver failure. Cytometry A 2012; 81: 823-34.

23. Xiu F, Stanojcic M, Diao L, Jeschke MG. Stress hyperglycemia, insulin treatment, and innate immune cells. Int J Endocrinol 2014; 2014: 486403. (Review)

24. Kumar P, Rao GN, Pal BB, Pal A. Hyperglycemia-induced oxidative stress induces apoptosis by inhibiting PI3-kinase/Akt and ERK 1/2 MAPK mediated signaling pathway causing downregulation of 8-oxoG-DNA glycosylase levels in glial cells. Int J Biochem Cell Biol 2014; 53: 302-19.

25. Komiya N, Hirose H, Saisho Y, Saito I, Itoh H. Effects of 12-month valsartan therapy on glycation and oxidative stress markers in type 2 diabetic subjects with hypertension. Int Heart J 2008; 49: 681-9.

26. Rogacev KS, Cremers B, Zawada AM, et al. CD14++CD16+ monocytes independently predict cardiovascular events: a cohort study of 951 patients referred for elective coronary angiography. J Am Coll Cardiol 2012; 60: 1512-20.

27. Montange D, Davani S, Deschaseaux F, et al. The number of circulating CD14 (+) cells is related to infarct size and post infarct volumes in ST segment elevation myocardial infarction but not non-ST segment elevation myocardial infarction. Exp Clin Cardiol 2012; 17: 131-5.

28. Ziegler-Heitbrock L. The CD14+ CD16+ blood monocytes: their role in infection and inflammation. J Leukoc Biol 2007; 81: 58492. (Review)

29. Belge KU, Dayyani F, Horelt A, et al. The pro-inflammatory CD14+CD16+DR++ monocytes are a major source of TNF. J Immunol 2002; 168: 3536-42.

30. Hristov M, Leyendecker T, Schuhmann C, et al. Circulating monocyte subsets and cardiovascular risk factors in coronary artery disease. Thromb Haemost 2010; 104: 412-4.

31. Liu Y, Imanishi T, Ikejima H, et al. Association between circulating monocyte subsets and in-stent restenosis after coronary stent implantation in patients with ST-elevation myocardial infarction. Circ J 2010; 74: 2585-91.

32. Cancellieri C, Caronni N, Vacchini A, et al. Review: Structurefunction and biological properties of the atypical chemokine receptor D6. Mol Immunol 2013, 55: 87-93. (Review)

33. Heine GH, Ulrich C, Seibert E, et al. CD14(++) CD16+ monocytes but not total monocyte numbers predict cardiovascular events in dialysis patients. Kidney Int 2008; 73: 622-9.

34. Berg KE, Ljungcrantz I, Andersson L, et al. Elevated CD14++ CD16- monocytes predict cardiovascular events. Circ Cardiovasc Genet 2012; 5: 122-31.

35. Hilgendorf I, Swirski FK. Making a difference: monocyte heterogeneity in cardiovascular disease. Curr Atheroscler Rep 2012; 14: 450-9. (Review)

36. Spahn JH, Kreisel D. Monocytes in sterile inflammation: recruitment and functional consequences. Arch Immunol Ther Exp (Warsz) 2014; 62: 187-94.

37. Braesch-Andersen S, Paulie S, Smedman C, Mia S, KumagaiBraesch M. ApoE production in human monocytes and its regulation by inflammatory cytokines. PLoS One 2013; 8: e79908.

38. Luis SA, Yamada A, Khandheria BK, et al. Use of three-dimensional speckle-tracking echocardiography for quantitative assessment of global left ventricular function: a comparative study to three-dimensional echocardiography. J Am Soc Echocardiogr 2014; 27: 285-91.

\section{Supplemental Files}

Supplemental Table I, II

Supplemental Figure 1,2

Please find supplemental files;

https://www.jstage.jst.go.jp/article/ihj/56/1/56_14-174/_article/supplement 